\title{
End-user experience with the SCoTSS Compton imager and directional survey spectrometer
}

\author{
Laurel E. Sinclair ${ }^{a, b}$, Andrew McCann ${ }^{a}$, Patrick R.B. Saull ${ }^{c, b}$, Nathan \\ Murtha ${ }^{\mathrm{a}, \mathrm{c}, \mathrm{b}}$, Rodger L. Mantifel ${ }^{\mathrm{c}}$, Christian V.O. Ouellet ${ }^{\mathrm{c}}$, Pierre-Luc \\ Drouin' $^{g}$, Audrey M.L. Macleod ${ }^{\mathrm{c}}$, Brian Le Gros ${ }^{\mathrm{d}}$, Ian Summerelle ${ }^{\mathrm{e}}$, Jens H. \\ Hovgaard $^{f}$, Stephen Monkhouse ${ }^{\mathrm{f}}$, Flaviu Stanescu ${ }^{\mathrm{f}}$, Guy Jonkmans ${ }^{\mathrm{g}}$ \\ ${ }^{a}$ Canadian Hazards Information Service, Natural Resources Canada \\ ${ }^{b}$ Department of Physics, Carleton University \\ ${ }^{c}$ Measurement Science and Standards, National Research Council Canada \\ ${ }^{d}$ Canada Border Services Agency \\ ${ }^{e}$ Royal Canadian Mounted Police \\ ${ }^{f}$ Radiation Solutions Inc. \\ ${ }^{g}$ Defense Research and Development Canada
}

\begin{abstract}
The Silicon photomultiplier-based Compton Telescope for Safety and Security (SCoTSS) has been developed incorporating end-user requirements into the design process. The end-user group includes those responsible for mobile survey in the event of a radiological or nuclear accident, those responsible for radiation survey support to security operations at major events and at Canadian borders, as well as some of those responsible for Canadian defense applications. The SCoTSS development program has reached a technology readiness level of eight, and we are proceeding with field trials of the instrument in highfidelity operational environments. Prospective end users have been involved in trial set up and execution, assuring applicability in their mission spaces. SCoTSS has been subject to trials involving hidden sources, heavily shielded sources, imager moving with respect to source, and complicated man-made surroundings. Our operators value high sensitivity for anomaly geolocation and mapping. End users also require an instrument which is capable of direction reconstruction in motion, as well as rapid imaging of a field of view. We have developed a "time to image" measure which allows for quantitative comparison of imagers of fundamentally different technology, where one design may have an advantage in terms of energy resolution and compactness and another
\end{abstract}

Email addresses: laurel.sinclair@canada.ca (Laurel E. Sinclair), andrew.mccann@canada.ca (Andrew McCann)

Full article appears in Nucl. Instrum. and Methods Phys. Res. A, 161683 
design may have an advantage in terms of efficiency and cost effectiveness. We present here the performance of the SCoTSS imager in rapid direction finding. As well, we compare the time to image quantity for the SCoTSS imager and the H3D Polaris-H Quad imager where the data were taken under equivalent conditions. This quantitative measure of imaging performance can allow operators to make an informed choice of the design that meets their needs taking into consideration also weight and size as well as budgetary constraints.

Keywords: Compton imaging, Compton telescope, Scintillator, Security, Radiation detection

\section{Introduction}

The Silicon photomultiplier-based Compton Telescope for Safety and Security, SCoTSS, is the first Compton imager based on solid scintillators coupled to silicon photomultiplier ( $\mathrm{SiPM}$ ) optical sensors [1]. SCoTSS is composed of pixelized layers of crystal scintillator in a modular configuration allowing users to specify the mass required for their operations. SCoTSS has been designed for use in radiological and nuclear incident investigations, nuclear non-proliferation applications, and to support safety and security activities in Canada. A full nine-module imager in a $3 \times 3$-module configuration employs a total scintillator mass of over $15 \mathrm{~kg}$. Thus, the $3 \times 3$-module version of SCoTSS can act as a survey spectrometer with the sensitivity to do isotope identification and alarming at dose rates consistent with background levels in sub-second acquisitions. Such sensitivity enables mobile survey operations in total-count mode with real-time alarming, isotope identification, and geo-location. In addition, SCoTSS can act as a directional survey spectrometer, with source localization anywhere within the entire $4 \pi$ solid angle based on real-time self-shielding indicators. Once a radiation field of interest has alarmed, the operator can switch from isotope alarming mode to targeting specific fields and directions for gamma-ray imaging. A smaller single-module version of the SCoTSS imager can be utilized where compactness is a priority for hand-held or backpack use.

In this work we review the performance of the SCoTSS $3 \times 3$-module imager with specific reference to the requirements of operational deployment. Several other gamma imagers are now available commercially, some also based on Compton imaging [2, 3], and some on coded-aperture or pinhole mask techniques [4]. Security and emergency response operators require quantification of imager performance in order to choose the optimum device for their operations. We define a quantity called "time-to-image" which can be used to 
quantitatively compare the performance of any imager designs, provided data are acquired under equivalent conditions. Data under equivalent conditions have been acquired for the SCoTSS $3 \times 3$-module imager and single-module imagers and for the commercially available H3D Polaris-H Quad Compton imager [5]. These three devices will be compared herein. Other groups can reproduce these experimental conditions in the future and calculate time-toimage as well to bring more imagers into the comparison.

\section{The SCoTSS Instrument}

\subsection{Hardware Design}

The parameters of the final design of the SCoTSS imager were chosen after several years of prototyping and simulation activity [6, 7]. Crystal scintillator was chosen as the active medium in order to be able to provide the sensitivity and field-worthiness of standard gamma-ray mobile survey and mapping instruments currently in use by radiological-nuclear security teams. In specific, thallium-doped cesium iodide was chosen for its ease of use and for the correspondence of its optical emission spectrum to the spectral response of the SensL [8] silicon photomultipliers. The SCoTSS imager consists of two segmented layers. An array of cubic crystals, each $1.35 \mathrm{~cm}$ on a side define the front face of the detector. The parameters of this front "scatter" layer were optimized to ensure Compton scatter interactions yield excellent positional and energy resolution, while minimizing the probability of multiple scatters within the layer. The second "absorber" layer, an array of cubic CsI(Tl) crystals $2.8 \mathrm{~cm}$ on a side, has been optimized to absorb all of the remaining energy of

gamma rays scattering in the front layer while achieving the required level of positional resolution. Each SCoTSS crystal is individually mated to an SiPM sensor and optically isolated.

The SCoTSS instrument employs a modular design, with a single module consisting of a $4 \times 4$ array of crystals in both the scatter and absorber layers. This design allows for different configurations of SCoTSS modules to be combined and arranged depending on what is suitable and practical in a given deployment setting.

Figure 1 (a) shows the $3 \times 3$-module SCoTSS imager discussed here. It employs a $3 \times 3$ configuration of single modules, resulting in $12 \times 12$ arrays of measurement channels in both the scatter and absorber layers.

Readout of the channels is done by custom-designed coincidence-timing and digitizing electronics connected to the SiPM sensors. An optical camera, positioned behind a transparent panel in the front of the imager enclosure, enables overlay of gamma-ray and optical images. Inside the carbon-fibre 


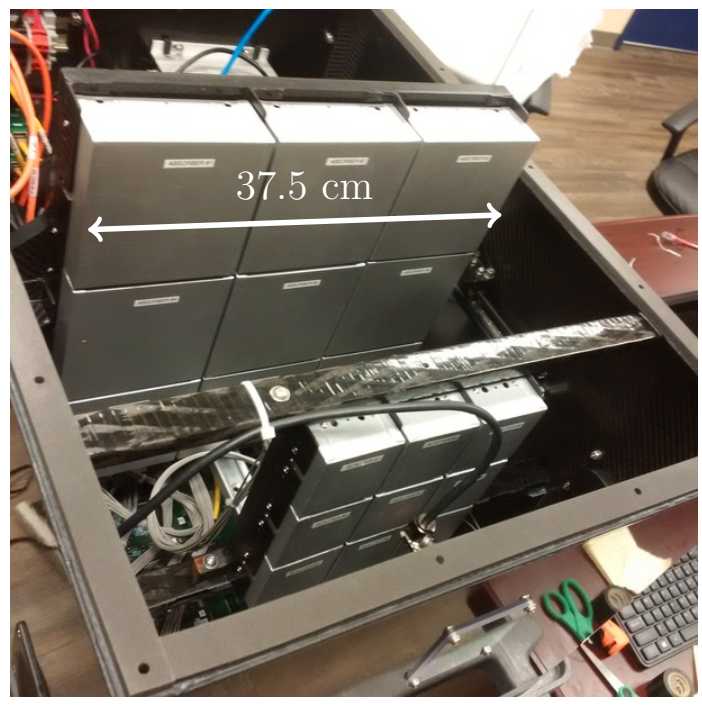

(a) SCoTSS Imager

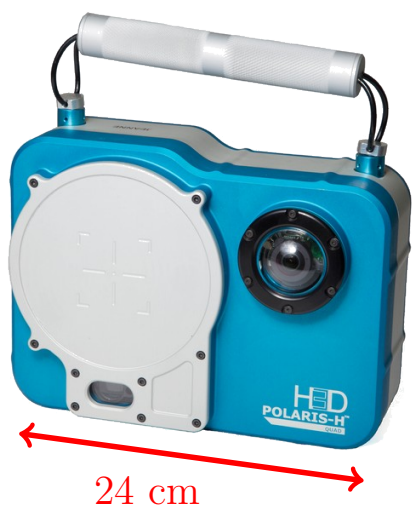

(b) H3D Polaris-H Quad Imager

Figure 1: Photographs of the imagers. (a) $3 \times 3$-module SCoTSS Imager with the top panel of the enclosure removed. The $3 \times 3$ arrays of single SCoTSS modules are visible. The smaller array is the scatter layer and the larger array is the absorber layer. A dimension of $37.5 \mathrm{~cm}$ across the absorber layer is indicated. This is an irreducible dimension for this design whereas the exterior housing is still under development. (b) H3D Polaris-H Quad commercially available imager with indication of longest exterior dimension.

enclosure, the scatter and absorber layers are attached to rails which allow the distance separating the layers to be adjusted. This "nuclear zoom" feature allows for an initial low resolution wide-angle field of view image to be followed by a higher precision image over a narrower field of view. For this study the inter-plane distance in both simulation and data was set to $20 \mathrm{~cm}$ and $8 \mathrm{~cm}$ for the $3 \times 3$-module and single-module imagers respectively.

The SCoTSS imager in a $3 \times 3$ configuration is suitable for truckborne mobile survey applications. A single module can also be assembled for handheld or backpack deployments. The Polaris-H Quad imager is a commercially available imager from $\mathrm{H} 3 \mathrm{D}$ which is also suitable for hand-held application. It is pictured in Figure 1 (b). The Polaris-H Quad imager utilizes cadmium zinc telluride semiconductor technology and weighs $3.5 \mathrm{~kg}$.

\subsection{Sensitivity and Energy Resolution}

Figure 2 shows the average energy spectrum in counts per second measured by the $3 \times 3$-module and single-module SCoTSS imagers when exposed to a 
$1 \mathrm{mCi}$ Cs-137 source located at a distance of $10 \mathrm{~m}$. Evident in Figure 2 is

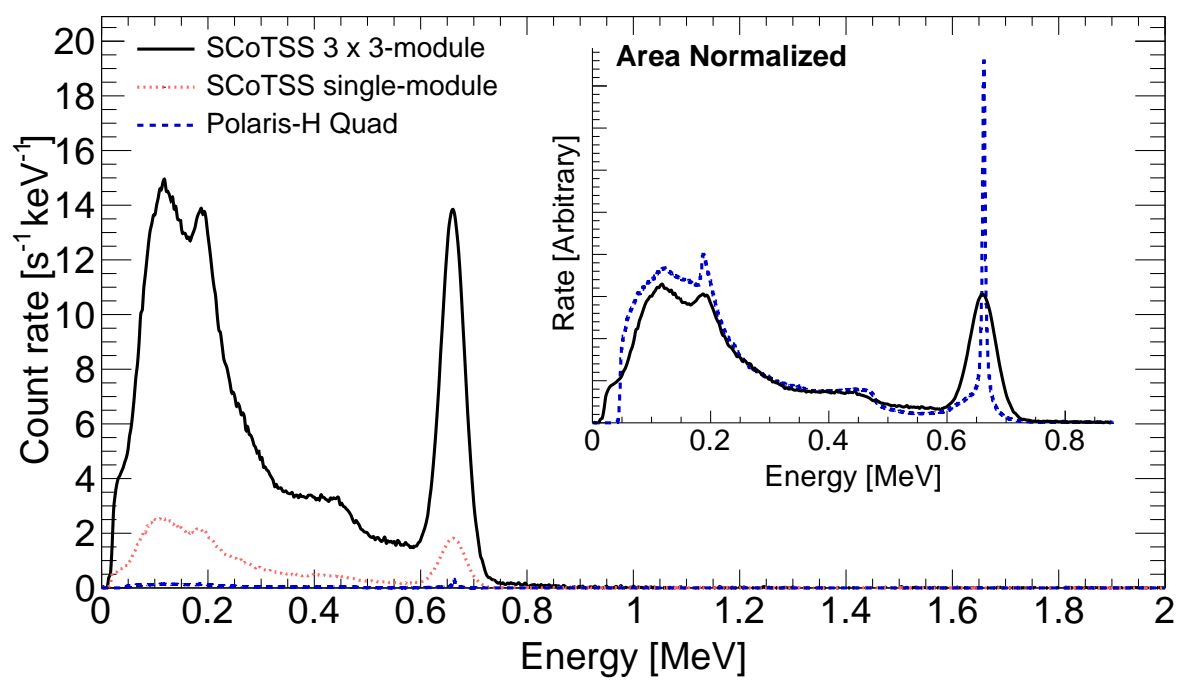

Figure 2: Energy spectra measured by the SCoTSS and the H3D Polaris-H Quad imagers when exposed to a gamma-ray flux equivalent to that emitted by a $1 \mathrm{mCi}$ Cs-137 source located at a distance of $10 \mathrm{~m}$ for one second. (Multiple one second spectra were averaged to produce these smooth distributions.) The insert, zoomed over the $0-0.88 \mathrm{MeV}$ energy range, shows the spectra areanormalized and serves to illustrate the relative energy resolution of the two instruments. Some small high-energy features visible in the SCoTSS spectrum are due to imprecise background subtraction. The Polaris-H Quad spectrum was accumulated using a $5 \times$ stronger field and scaled down for the comparison, so natural background contributions to the Polaris-H Quad spectrum are considered to be negligible.

the $662 \mathrm{keV}$ gamma-ray emission line from Cs-137, illustrating the $\sim 7 \%$ fullwidth at half maximum (FWHM) energy resolution of the SCoTSS imager at that energy. The single-module SCoTSS imager accumulates a few counts per second in the photopeak for this source which gives a dose rate at the detector of only $15 \mathrm{nGy} / \mathrm{hr}$, less than the natural background. The $3 \times 3$ module SCoTSS detector on the other hand accumulates around a hundred counts in the photopeak in one second. A one-second sampling rate is the typical rate used when performing high sensitivity mobile radiometric survey and mapping [9]. Thus, the high sensitivity of the $3 \times 3$-module SCoTSS imager allows for isotope identification and alarming of sources at background levels during truckborne survey.

The Cs-137 energy spectrum measured by the H3D Polaris-H Quad instru- 
ment under equivalent conditions is also plotted in Figure 2 allowing a clear comparison of the sensitivity. The Polaris-H Quad detector accumulates less than one count in the photopeak per second under these conditions, thus it would require a much longer time of acquisition than either SCoTSS detector in order to detect this source at this distance.

The inset figure in Figure 2 shows the energy spectra of the SCoTSS $3 \times$ 3 -module and Polaris-H Quad imagers area normalized. This allows for a comparison of the shapes of the spectra and it is clear that the cadmium zinc telluride detector used in Polaris-H Quad provides a superior energy resolution. Polaris-H Quad is quoted to achieve $\leq 1.1 \%$ FWHM at $662 \mathrm{keV}[10$. There may be circumstances such as environments cluttered with many radioactive sources of different energies of emission in which superior energy resolution would provide improved imaging capability. Exploration of these effects will be the subject of future work.

The self-shielding of the crystal configuration in the SCoTSS imagers can be utilized to calculate a direction to a source from any incoming angle, without requring a Compton coincidence and without restriction to a field of view. The sensitivity of the $3 \times 3$-module imager means that these directions can be calculated for relatively weak sources on a second by second basis. Thus, from an operational standpoint, the $3 \times 3$-module SCoTSS can be deployed in a moving vehicle (truck, helicopter, etc) which can survey a region and alarm when in the field of a source of interest with a real time directional indicator that localizes the direction of incoming radioactivity in $4 \pi$.

\subsection{Real-time display}

Figure 3 (a) shows an example of the screen available to the SCoTSS operator in real time. The upper left windows shows a "waterfall" plot. One-second energy spectra are displayed with energy on the x-axis and time on the y-axis. The number of counts in each spectrum is indicated by the colour scale. The lower left subwindow shows a total deposited energy spectrum, summed over all detector channels. By default the current energy spectrum accumulated in one second intervals is shown. Alternately (as shown here), the user may select a region of time of interest on the waterfall plot, and then the lower left subwindow will show the energy spectrum for that time period.

The right subwindow shows the image from the optical camera with the contours of the gamma image overlain. The operator has selected an energy band of interest from the waterfall plot and it is this energy band for which the Compton image has been reconstructed. Thus, it is not necessary for the operator to know a priori the identity of the radioactive material of interest in order to image it. A white circle (difficult to see against the sky in this image) 
also indicates the result of an independent assessment of source location based on simple self-shielding direction finding using five seconds of data.

This particular image was grabbed from the SCoTSS operator laptop screen during an operational exercise. The source which is being imaged had not yet been deployed. It was situated within its heavily shielded shipping container in a trailor well to the right side just within the imager's field of view. The source was about $30 \mathrm{~m}$ away, on the opposite side of the parking lot from the location of the imager.

Figure 3 (b) shows a sequence of directions reconstructed using the selfshielding information during an operational exercise. The directions have been plotted in absolute coordinates. The tail of each vector is located at the position of the survey vehicle containing the imager and the vectors indicate the compass direction to the source from that location. From the standpoint of an operator, this rapid compass direction which allows for optimal positioning of the survey vehicle is highly valuable in addition to the information contained within the Compton images.

\section{Methods}

\subsection{Self-shielding and directionality}

Though designed as a Compton imaging telescope, the SCoTSS imager can be considered as a close-packed array of self-shielding elements. Self-shielding directional methods use the relative rates of energy deposits in each detector element to determine the most likely direction to the source of emission. Direction-finding results for a single-module imager making use of a lookup table method have been presented previously [11]. Here we will present direction-finding results for the $3 \times 3$ module imager using a method which we call Simple Direction Finding (SDF).

Some of the self-shielding properties of the $3 \times 3$-module SCoTSS imager are presented in Figure 4 where the coordinate system and angle definitions are shown in Figure 5. Figure 4 shows the relative count-rates of each crystal in the $12 \times 12$ channel array of the absorber layer for various positions of a Cs-137 point source as determined through Geant4 [12] simulations. In Figure 4 (a) the source is at the front of the imager slightly above the horizon. The scatter array thus casts a shadow on central channels of the absorber layer. In Figure 4 (b) the source is $40^{\circ}$ above the horizon, and the shadow of the scatter plane on the absorber plane is that much lower. Figure 4 (c) shows the source edge-on to the absorber layer and the strong shielding of the entire layer results in straightforward reconstruction of the source direction in this case. In Figure 4 (d) the source is again toward the side but the source may 
be reconstructed to be offset somewhat from the edge since count rates are consistent with gamma rays also incident on the face of the absorber layer.

The SDF method starts by choosing three perpendicular axes $(\hat{u}, \hat{v}, \hat{w})$ in the detector geometry, e.g. "top-bottom", "left-right" and "back-front". The difference in the measured rate projected along these directions is then computed, resulting in a three-dimensional vector. If constructed sensibly, this vector provides a crude estimate of the direction of the source of emission. The SDF algorithm as applied to the SCoTSS $3 \times 3$-module imager is described in Figure 6. The geometry of the detector is first simplified by grouping the rates in the scatter plane into 4 quadrants. The same is done for the absorber plane, reducing the number of rates from 288 to eight. The $\hat{u}$ and $\hat{v}$ directions were chosen to lie in the $x y$-plane as defined in Figure 5, but with a $45^{\circ}$ rotation about the $z$-axis. The $\hat{w}$ direction is parallel to the "back-front" direction of the SCoTSS geometry which also lies parallel to the $z$-axis as defined in Figure $5(\hat{w} \equiv \hat{z})$. Due to the difference in size and mass between the scatter and absorber planes a scale factor weighting the scatter rates, $\alpha$, is introduced when projecting the rate-difference along the $\hat{w}$ direction. For the current study we set $\alpha \equiv 6.8$. Using the standard Cartesian-to-spherical-polar conversions (equation line 4 in Figure 6) the SDF vector is converted into the polar and azimuthal angles defined in Figure 5.

\subsection{Compton Imaging}

Compton imaging methods require coincident energy depositions, reconstructing the directional information of only those impinging gamma rays which scatter in the first layer and are fully absorbed in the second. Though this requirement reduces the number of considered detections to $\sim 0.5 \%$ of the total number incident for mid-range energies of $\sim 662 \mathrm{keV}$, those which meet the selection criteria contain a high quality of directional information. The incident direction of each detection is known to lie on the surface of a cone projected outward from the detector along the axis connecting the scatter and absorption interaction points. Successive cones "back-projected" outward from the detector intersect and, when overlayed, form an image of the locations of emission. In the case of a single point source, only three Compton coincidence events are needed to uniquely define the location of the source within measurement uncertainty. Assuming a point source, a $\chi^{2}$-minimization algorithm can be applied to the back-projected Compton cones to find the direction most consistent with all cones [6]. This method allows for rigorous treatment of the measurement uncertainty on the individual cones in the reconstruction, permitting a source position measurement with high precision and accuracy. 
To quantify the point-source localization performance of an instrument or algorithm, we propose a metric called "time-to-image", which is the reconstruction precision obtained after a certain acquisition time [13]. The reconstruction precision is formally the root mean square (RMS) spread of reconstructed Compton image directions computed from independent datasets of a certain acquisition time. Generally, as the acquisition time increases, greater and greater angular precision is achieved. The basic instrument characteristics: Doppler broadening, efficiency, sensitivity, energy and angular resolution, combine and affect the time-to-image metric, which can be seen as a global performance metric. Detector design optimized for any one or any set of the basic instrument characteristics, at the expense of others, can degrade the time-toimage performance. Since the time-to-image metric can be constructed for any instrument or algorithm which can localise a point source it can be used to compare the performance of detectors which may each have superiority in one or more of the individual performance measures.

\section{Results}

\subsection{Self-shielding and directionality}

The SCoTSS detector has a natural coordinate system as shown in Figure 5 , with a pole along the symmetry axis connecting the centres of the scatter and absorber planes. In the natural coordinate system there is a polar angle with respect to this pole, and an azimuthal angle about it. As demonstrated in Figure 4, there is plenty of information in the pattern of energy deposits from the inclusive (non-coincident) trigger, for an accurate reconstruction of the azimuthal angle of a source direction about the symmetry axis. That being said, the $3 \times 3$-module SCoTSS detector configuration was not optimized for self-shielding directionality indicators. For example, Geant4 simulations (not shown) have shown that when the scatter and absorber planes are separated by $20 \mathrm{~cm}$, the detector responses due to sources located at polar angles near $60^{\circ}$ and $120^{\circ}$ are practically indistinguishable. In these locations, neither plane shields the other from the incoming radiation and the self-shielding pattern within the planes cannot break the degeneracy in any reasonable length of time. Note however that these simulations of the $3 \times 3$-module SCoTSS detector neglected much of the passive detector hardware which may create shielding effects sufficient to break or appreciably mitigate these degeneracies. This will be exploited in future work.

In any case, neither the polar nor the azimuthal angle with respect to the natural SCoTSS coordinate system are of primary importance in operations. The angle of primary use in operations is the bearing angle, or the compass 
direction to the source location from the detector's current position. The bearing angle guides the vehicle operator closer to the source and enables the operator to bring the source within the imager's field of view. Relative bearing comes from the projection of the direction vector into the $x z$-plane, the plane of the earth neglecting topography and curvature. The relative bearing angle depends on both the azimuthal and polar angles, as shown in Figure 5. Absolute bearing angle is then calculated by adding the heading of the vehicle carrying the imager to the relative bearing angle.

The directional response of the $3 \times 3$-module SCoTSS imager to a $10 \mathrm{mCi}$ Cs-137 point source located at a distance of $10 \mathrm{~m}$ was calculated using the Geant4 simulation package. Multiple trials were simulated at various polar and azimuthal directions each with one second of data. From each data set a relative bearing in the $x z$-plane was reconstructed. Figure 7 shows the SDF reconstructed relative bearing against true relative bearing for these one second trials. The ensemble of reconstructed and true pairs is plotted using a profile histogram showing the mean and spread in each bin (black circles). We find that, even for just a one-second acquisition period, there is a rough correspondence between the reconstructed and true relative bearings. Poor correlation arises in some regions largely due to degeneracy of the self-shielding detector response for sources at some polar angles as mentioned previously.

Self-shielding information can be calculated from every energy deposit, without having to wait for a good Compton coincidence event. Thus, the SDF direction to the source can be mapped while the instrument is in motion, even for relatively weak sources. The utility of this angle in a live-agent field exercise was illustrated by Figure 3 (b). Despite the regions of poor correlation shown in Figure 7 around relative bearing of $200^{\circ}$, the bearing angle information is of great use operationally. It provides a first clue for operators as to which side of the vehicle a threat agent is located on. This allows for orientation of the imager with the source within the field of view, and rapid Compton imaging.

\subsection{Compton imaging}

In Figure 8 the time-to-image metrics of the SCoTSS single-module and $3 \times 3$-module imagers and of the H3D Polaris-H Quad imager when exposed to a gamma-ray flux equivalent to that emitted by a $1 \mathrm{mCi}$ Cs-137 point source located at a distance of $10 \mathrm{~m}$ are shown. All three imagers exhibit a precision of localization which improves rapidly with increasing acquisition time. The $3 \times 3$-module SCoTSS imager achieves a localization precision of better than $2^{\circ}$ in under 3 seconds. This points to the possibility to make use of the $3 \times 3$-module SCoTSS imager not only for isotope alarming, identification and direction-finding in motion, but also for imaging in motion. In contrast to 
the $3 \times 3$-module imager, the single-module hand-held SCoTSS imager would require longer, approximately one minute to achieve the same image precision. Despite its superior energy resolution, the H3D Polaris-H Quad imager requires even longer, over $\sim 2$ minutes, to achieve this image precision. Since operators who require a handheld imager probably do not also wish to carry a tripod it is worth pointing out that sufficiently rapid imaging using a handheld imager is only going to be possible for much higher activity sources, or unshielded sources.

The importance of the comparison in Figure 8 is in the demonstration of a quantitative comparison of the performance of detectors of radically different design. Although some of the designs under comparison have superior efficiency and others have superior angular resolution measure and energy resolution, it is nevertheless possible to compare their overall performance quantitatively, at least for the case of reconstruction of a point source. Future presentations of imager performance should include this measure, to allow an operator to choose between sensitivity and volume or cost, according to his or her mission space.

\section{Discussion and Conclusion}

Characteristics of a Compton imager based on crystalline scintillator and read out with silicon photomultipliers have been presented previously by this group. What is new in this study is that we report on the imager's performance from the particular perspective of a community of responder end users. Mobile surveyors value the capability to map the second by second counts in energy windows. For this they require an imager with not only georeferenced spectra but sufficient sensitivity for detection and alarming based on the information gained in one second of acquisition while moving. Operators also value obtaining an indication of the direction to the source in real time while in motion. For an imager, this again means very high sensitivity as well as omni-directional acceptance. The self-shielding information any imager possesses can be used to rapidly produce an approximate direction to the hottest source in the full $4 \pi$ solid angle.

It can be difficult for operators to choose among detectors of differing technologies when for example one detector provides superior energy resolution while another detector has superior efficiency. Operators may have to choose between energy resolution and sensitivity as the materials which provide superior energy resolution tend to be too expensive to acquire in large quantities. Some operators nevertheless require the imaging cability to be provided in as small and light a package as possible. The time-to-image quantity discussed 
in this manuscript can provide a means for quantitative comparison of the overall performance of imagers which have different strengths on individual parameters. Publication of this measure by the various groups with imagers under development should be performed. Armed with this quantitative comparison of imager performance, operators could then balance the competing requirements for reduced size and complexity against improved efficiency and resolution for their particular mission space.

\section{Acknowledgements}

The authors are grateful to Dr. C. Wahl from H3D for helpful discussions and for provision of the data from the Polaris-H Quad detector shown herein. The authors gratefully acknowledge helpful discussions with a number of scientists from Defense Research and Development Canada. This work has been made possible with funding from Defense Research and Development Canada's Canadian Safety and Security Program. This is NRCan Contribution number 20180164.

\section{References}

\section{References}

[1] L. E. Sinclair, P. R. B. Saull, D. S. Hanna, H. C. J. Seywerd, A. M. L. MacLeod, and P. J. Boyle, "Silicon Photomultipler-Based Compton Telescope for Safety and Security (SCoTSS)," IEEE Trans. Nucl. Sci., vol. 61 No. 5, pp. 2745 - 2752, 2014.

[2] PHDS Co., 3011 Amherst Road, Knoxville, TN 37921, USA.

[3] H3D Inc., 812 Avis Dr., Ann Arbor, MI 48108, USA.

[4] CANBERRA, http://www.canberra.com/.

[5] C. G. Wahl, W. R. Kaye,W. Wang, F. Zhang, J. M. Jaworski, A. King, Y. A. Boucher, Z. He, "The Polaris-H imaging spectrometer" Nucl. Instr. and Meth. A, vol. 784 , pp. $377-381,2015$.

[6] L. E. Sinclair, D. S. Hanna, A. M. L. MacLeod, and P. R. B. Saull, "Simulations of a scintillator Compton gamma imager for safety and security," IEEE Trans. Nucl. Sci., vol. 56 Issue: 3, pp. 1262 - 1268, 2009. 
[7] P. R. B. Saull, L. E. Sinclair, H. C. J. Seywerd, P. J. Boyle, A. M. L. MacLeod, and D. S. Hanna, "A two-pixel Compton imager," Proc. SPIE, vol. 7665, pp. $76651 \mathrm{E}-76651 \mathrm{E}-11,2010$.

[8] sensL, Airport Business Park, Cork T12 CDF7, Ireland.

[9] "Airborne gamma ray spectrometer surveying," IAEA Technical Reports Series 323, 1991.

[10] H3D Inc, July 17, 2018, retrieved from https://h3dgamma.com/H400Specs.pdf

[11] A. McCann, A., L. E. Sinclair, P. R. B. Saull et al., "Which is better, a SCoTSS gamma imager, or an ARDUO UAV-borne directional detector?," arXiv:180\%.02052, presented at the 2017 IEEE Nuclear Science Symposium and Medical Imaging Conference, Atlanta, Georgia, U.S (2018).

[12] S. Agostinelli et al, "Geant4 - a simulation toolkit," Nucl. Instr. Meth. A, vol. 506, no. 3, pp. 250-303, 2003.

[13] P. R. B. Saull, L. E. Sinclair, H. C. J. Seywerd, D. S. Hanna, P. J. Boyle, and A. M. L. MacLeod, "First demonstration of a Compton gamma imager based on silicon photomultipliers," Nucl. Instr. and Meth. A, vol. 679, pp. 89 - 96, 2012. 


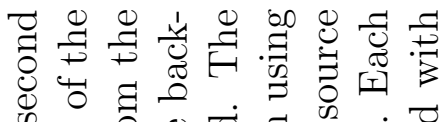

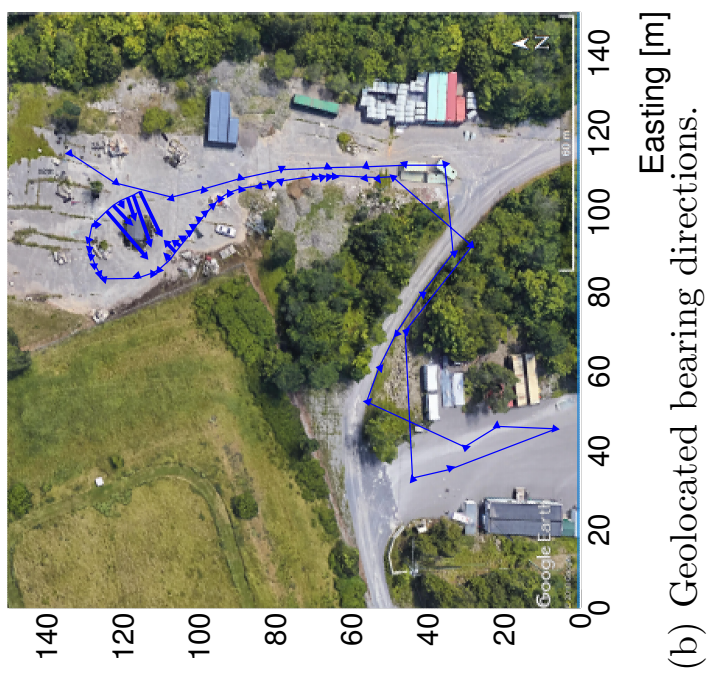

[w] Би!чนоN

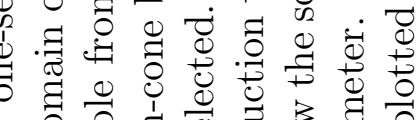

过若焉

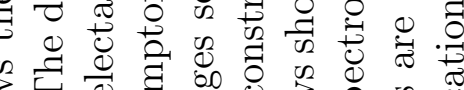

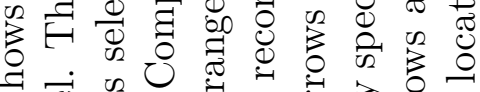

क बं

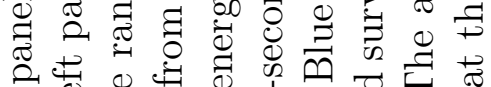

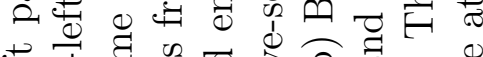

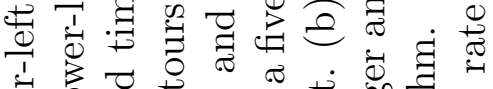

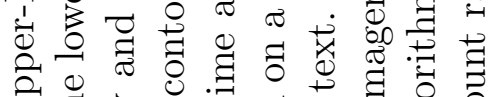

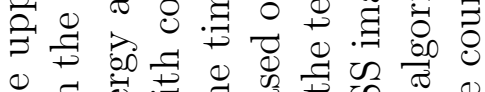

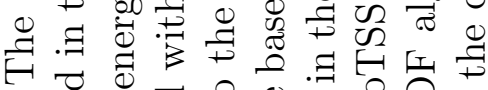

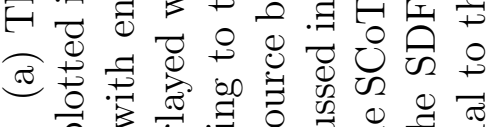

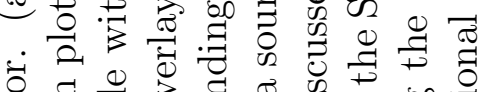

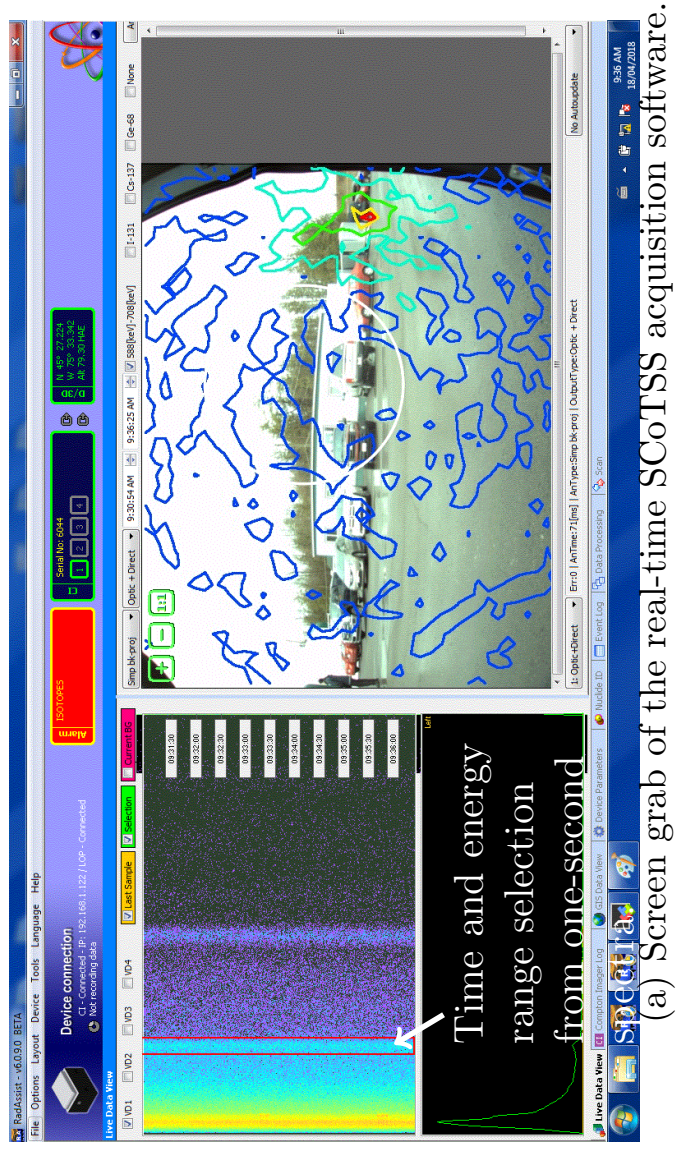

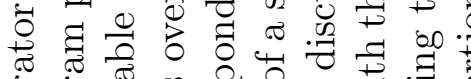

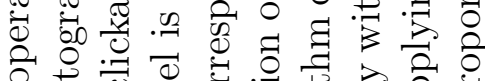

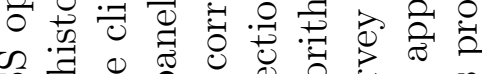

की

0 药

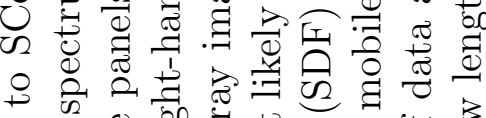

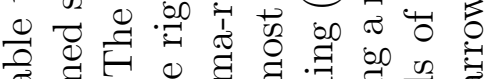

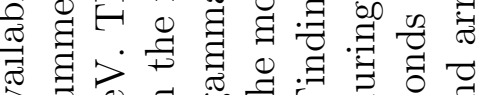

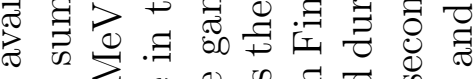

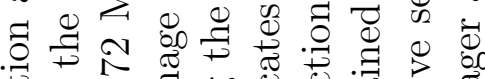

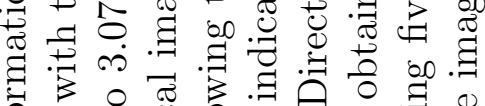

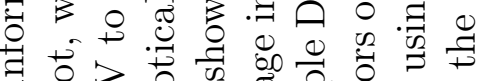

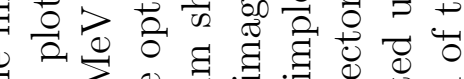

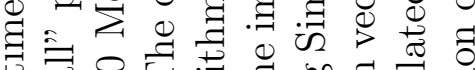
元 बే

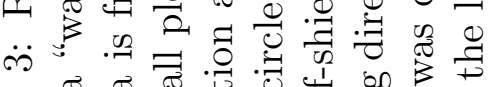

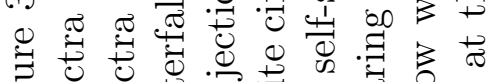

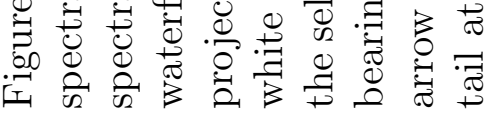




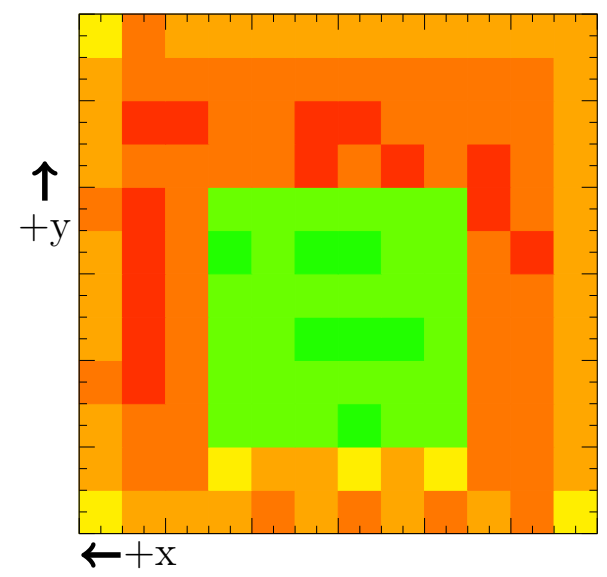

(a) Polar $170^{\circ}$, Azimuthal $90^{\circ}$

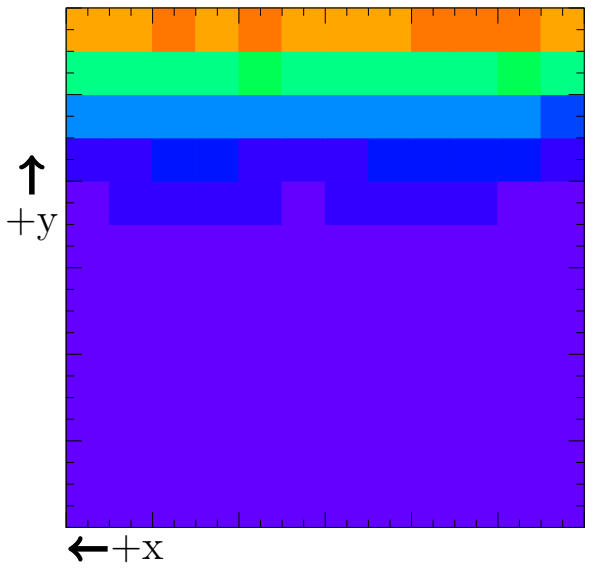

(c) Polar $90^{\circ}$, Azimuthal 90

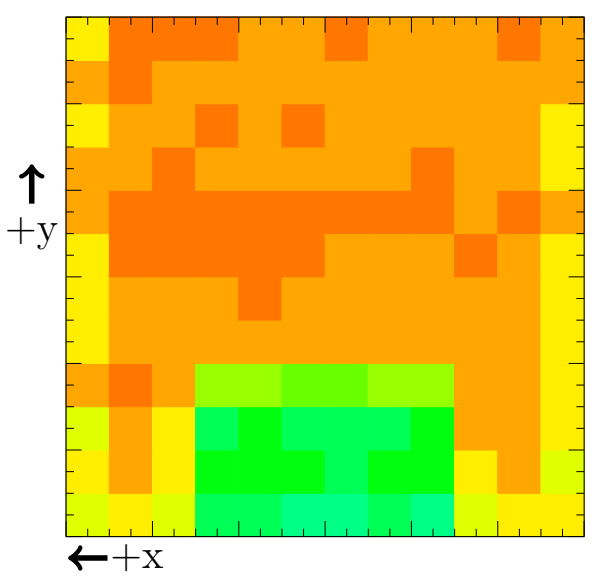

(b) Polar $140^{\circ}$, Azimuthal $90^{\circ}$

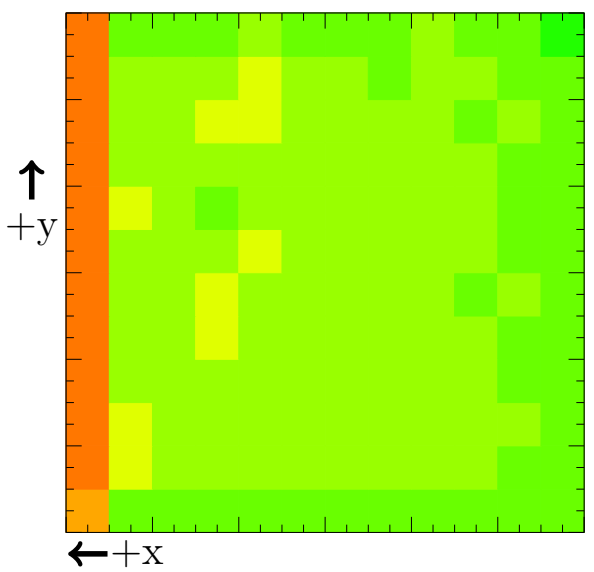

(d) Polar $60^{\circ}$, Azimuthal $0^{\circ}$ Low rate

High rate

Figure 4: Self-shielding properties of the SCoTSS imager. Plotted is the relative count rates in the $12 \times 12$ channel array of the SCoTSS absorber plane when a Cs-137 point source is located at the polar and azimuthal positions listed in the sub-captions. The low-rate regions in panels (a) and (b) indicate the shielding of the absorber plane by the material in the scatter plane. In panels (c) and (d), the source is located at the side of the detector, thus the material on the outer edges of the absorber shield the remaining material. Similar shielding effects occur in the scatter plane. Rates have been obtained from SCoTSS Geant4 simulations. Positive $z$-axis points into the page. See Figure 5 for a definition of angles and directions. 


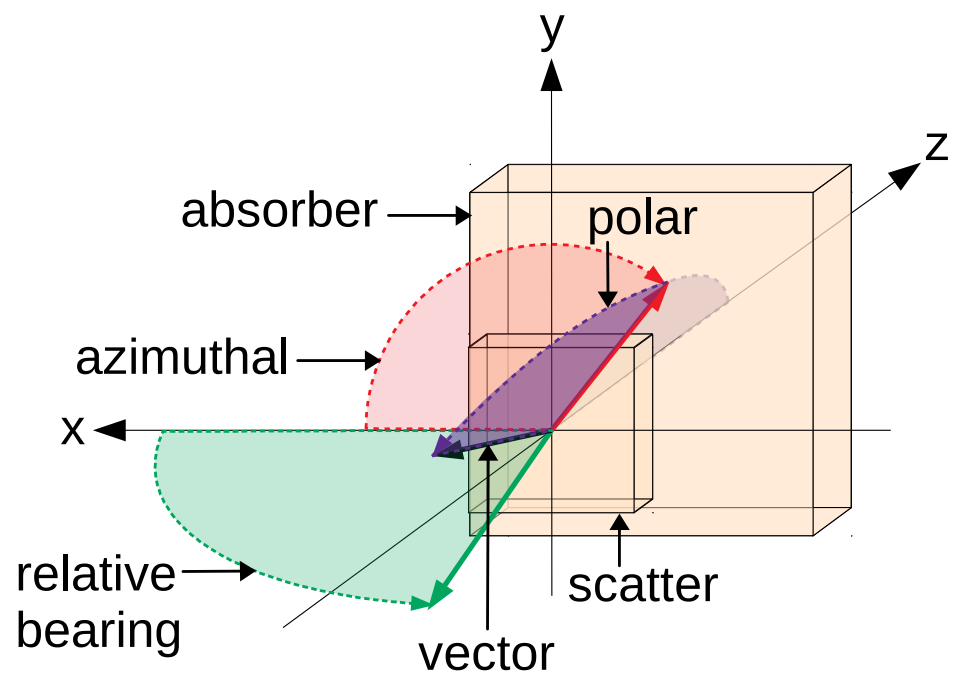

Figure 5: Schematic diagram of the SCoTSS imager and coordinate system. Directional vectors relative to an origin at the centre of the front face of the scatter layer are defined using spherical coordinates with a polar angle (drawn in purple) between the $z$-axis and the vector and an azimuthal angle (drawn in red) between the $x$-axis and the vectors projection on the $x y$-plane. It is useful to define the relative bearing angle between the $x$-axis and the vector's projection on the $x z$-plane (drawn in green). 


$$
\begin{aligned}
& \begin{array}{l}
\text { Scatter } \\
\begin{array}{|c|c|}
\hline S_{0} & S_{1} \\
\hline S_{2} & S_{3} \\
\hline
\end{array}
\end{array} \\
& \begin{array}{|}
\text { Absorber } \\
\hline A_{0} & A_{1} \\
\hline A_{2} & A_{3} \\
\hline
\end{array} \\
& u_{S}=\alpha\left(S_{0}-S_{3}\right) \quad u_{A}=A_{0}-A_{3} \\
& v_{S}=\alpha\left(S_{1}-S_{2}\right) \quad v_{A}=A_{1}-A_{2} \\
& u=u_{A}+u_{S} \\
& v=v_{A}+v_{S} \\
& w=\sum_{i=0}^{3}\left(A_{i}-\alpha S_{i}\right) \\
& \theta=\arccos \frac{w}{\sqrt{u^{2}+v^{2}+w^{2}}} \quad \phi=\arctan (v / u)-\pi / 4
\end{aligned}
$$

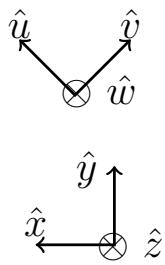

Figure 6: Simple direction finding algorithm applied to the SCoTSS $3 \times 3$ module imager. The $12 \times 12$ channels in the scatter plane are grouped into four quadrants and summed, resulting in a $2 \times 2$ signal array $S_{i=0,1,2,3}$. The same grouping is done in the absorber plane, resulting in the signal array $A_{i=0,1,2,3}$. The three-dimensional coordinate system $(\hat{u}, \hat{v}, \hat{w})$ is defined and the difference in the measured rates projected along these directions is computed, yielding the vector $(u, v, w)$ using the equations on lines 1,2 and 3. A scaling parameter, $\alpha$, is introduced to weight the rates in the scatter plane and account for the relative difference in size and stopping power of the scatter plane compared to the absorber. The polar angle, $\theta$, and azimuthal angle, $\phi$, as defined in Figure 5 are computed using the standard Cartesian-to-spherical-polar equations on line 4. 


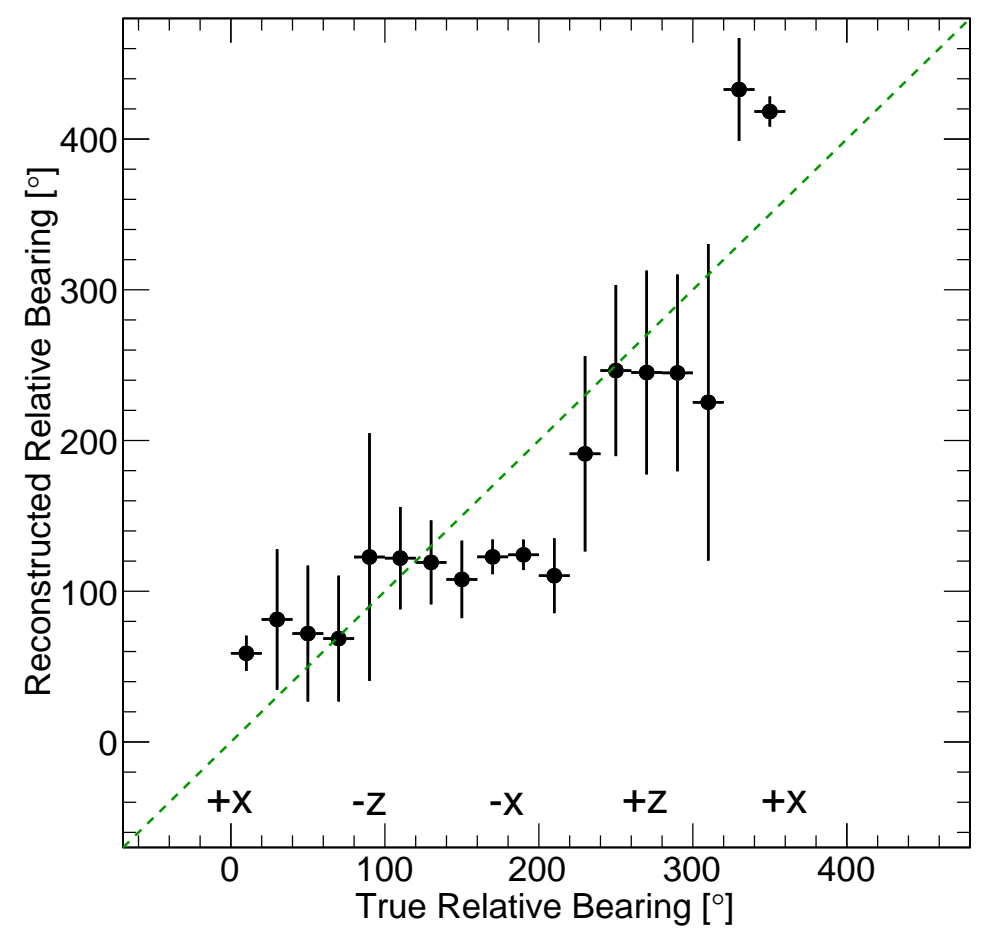

Figure 7: Reconstructed relative bearing from the self-shielding SDF algorithm applied to Geant 4 simulated data from the $3 \times 3$-module imager plotted against true relative bearing. The response of the $3 \times 3$-module SCoTSS imager to a $10 \mathrm{mCi}$ Cs-137 point source located at a distance of $10 \mathrm{~m}$ for trials of one second of data is shown. From each data set a relative bearing in the $x z$-plane is reconstructed, and the ensemble of reconstructed and true pairs is plotted using a profile histogram showing the mean and spread in each bin (black circles). The green dashed line indicates a perfect reconstruction $(y=x)$. See Figure 5 for a definition of angles and directions. 


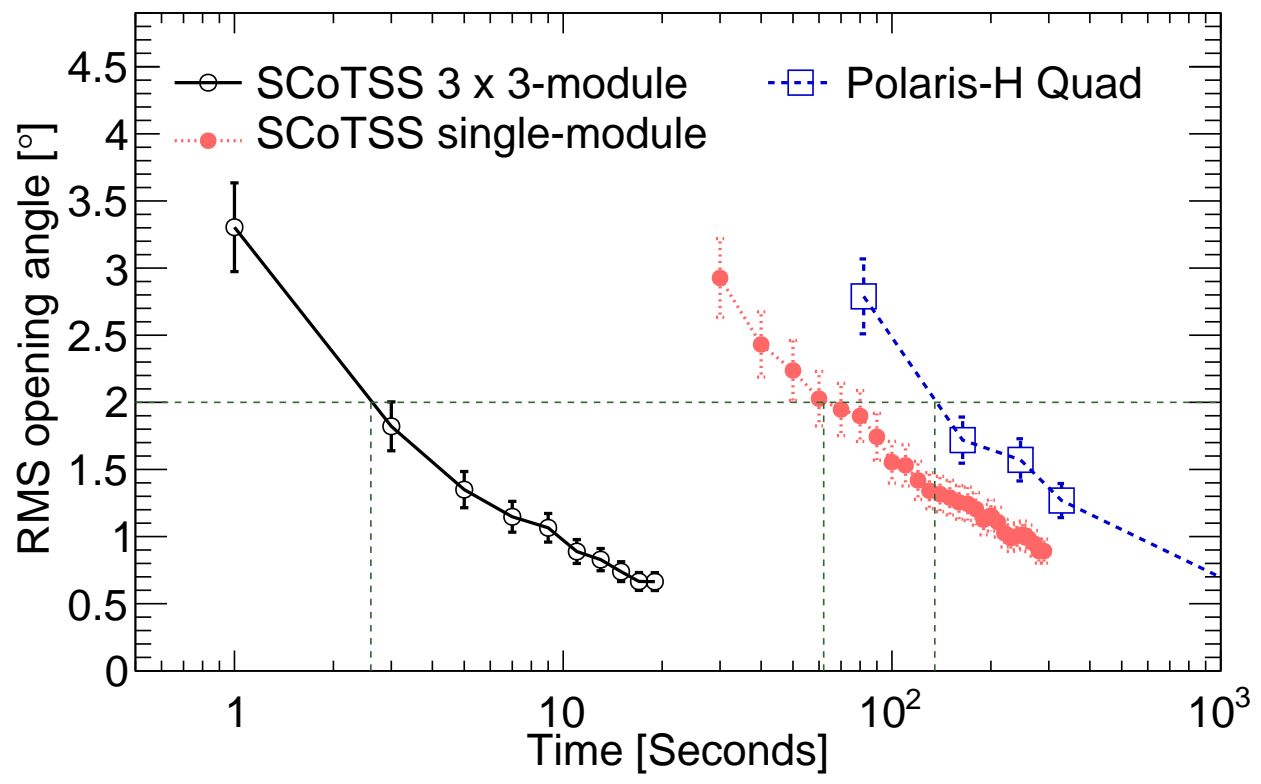

Figure 8: Source reconstruction precision as a function of acquisition time for the SCoTSS and the H3D Polaris-H Quad imagers when exposed to a gammaray flux equivalent to that emitted by a $1 \mathrm{mCi}$ Cs-137 point source located at a distance of $10 \mathrm{~m}$ and offset by $10^{\circ}$ from the negative $z$-axis (polar angle $170^{\circ}$ ). A localization precision within $2^{\circ}$ can be achieved in $\sim 4$ seconds with the SCoTSS imager and $\sim 2$ minutes with the H3D Polaris-H Quad imager as indicated by the vertical and horizontal dashed lines. Both data curves are assembled from real data acquired from the two imagers. The H3D Polaris-H Quad imager was exposed to a $5 \times$ higher actual field and the times were scaled up to the standard of comparison. The SCoTSS imager was exposed to a $0.5 \times$ lower actual field and the times were scaled down to the standard of comparison. 